\title{
Primary Frequency Response Reserve Products for Inverter-Based Resources
}

\author{
Manuel Garcia \\ Sandia National Laboratories \\ mgarc19@sandia.gov
}

\author{
Ross Baldick \\ University of Texas at Austin \\ baldick@ece.utexas.edu
}

\author{
Felipe Wilches-Bernal \\ Sandia National Laboratories \\ fwilche@sandia.gov
}

\begin{abstract}
Primary frequency control in power systems is being challenged by the large-scale integration of inverter-based resources (IBRs) because they do not typically respond to frequency fluctuations. This paper suggests introducing new reserve products into the electricity market that provide incentive for IBRs to contribute to primary frequency control in ways that take advantage of their fast-acting capabilities. In addition to a Primary Frequency Response (PFR) reserve product, which accommodates standard droop control, we suggest introducing a Fast Frequency Response (FFR) reserve product, a reserve product for Virtual Inertia (VI), which is also known as synthetic inertia, and an inertia product. We adopt a reserve requirement that guarantees sufficient primary frequency response reserve to adequately arrest frequency decline in response to a large generator outage within a certain margin. We place this reserve requirement into a real-time co-optimization problem, derive prices for each product and analyze the incentives provided to IBRs.
\end{abstract}

\section{Introduction}

The penetration of inverter-based resources (IBRs) has been increasing rapidly in modern power systems. These resources do not inherently provide inertial response and traditionally do not respond to frequency variations and thus they have placed a burden on primary frequency control. To provide incentive for IBRs to provide primary frequency control, this paper suggests introducing new reserve products into the electricity market. Reserve requirements are formulated and prices

This work was sponsored in part by the US Department of Energy Office of Electricity's Advanced Grid Modeling program.

The information, data, or work presented herein was funded in part by the Advanced Research Projects Agency - Energy (ARPA-E),

U.S. Department of Energy. The views and opinions of authors expressed herein do not necessarily state or reflect those of the United States Government or any agency thereof.

Sandia National Laboratories is a multimission laboratory managed and operated by National Technology \& Engineering Solutions of Sandia, LLC, a wholly owned subsidiary of Honeywell International Inc., for the U.S. Department of Energy's National Nuclear Security Administration under contract DE-NA0003525. are derived for these products. We then analyze the pricing incentives to determine which product an IBR may prefer to provide.

Various types of fast-acting primary frequency control have been proposed in the literature that are intended to be provided by IBRs [1]. This paper will focus on three such responses. Droop control responds proportionally to frequency deviations and is consistent with traditional primary frequency control used in power systems today. Traditional droop control is provided by synchronous generators and operates within a reserve type that we will refer to as Primary Frequency Response (PFR) reserve. Fast Frequency Response (FFR) exhibits a step response to the frequency deviation, fully activating when the frequency falls below a specified trigger point. Virtual Inertia (VI) is sometimes referred to as synthetic inertia and represents derivative control on the frequency, which is a response that is very similar to inertia provided by synchronous generators. In this paper a reserve product is defined for each of these types of primary frequency control in addition to a product for synchronous inertia. Each of these products are then coupled through an extended version of the reserve requirement from [2]. This reserve requirement is placed into a real-time co-optimization problem from which prices are derived for each product.

The Electric Reliability Council of Texas (ERCOT) is the first Independent System Operator (ISO) in North America to introduce an ancillary service product specifically intended to provide primary frequency control [3, 4]. For this reason, we focus our analysis on ERCOT; however, the models used in this paper are general and are applicable outside of Texas. Indeed, other regions have identified the need to address declining performance of primary frequency control, e.g. [5]. That being said, ERCOT's ancillary services market is currently being reformed and ERCOT has recently introduced Fast Frequency Response (FFR) reserve $[3,6]$. Two approaches have been taken to derive reserve requirements using ERCOT's definition of FFR reserve. The first was [7], which utilized empirically 
determined equivalency ratios. The second was $[2,8]$, which derived PFR reserve limits from first principles that place ramping rate limits on PFR reserve assigned to each generator based on total FFR reserve. Consistent with ISO protocols throughout the United States, these primary frequency response reserve requirements ensure frequency decline will be arrested before reaching some minimum frequency threshold in response to a large generator outage.

Electricity markets in the United States today have not yet introduced inertia or VI reserve as a product. In fact, reference [9] points out that there is no clear market structure that includes inertia and that this topic is an open research area. Very few works have proposed market structures that include inertia. The following references provide market mechanisms general enough to accommodate virtual inertia. Reference [10] provides a market mechanism based on auction theory that compensates inertia based on a Vickrey-Clarke-Groves payment rule. Reference [11] places a requirement into a dispatch problem and prices this requirement using marginal prices based on the Lagrange multipliers.

The requirement priced in [11] ensures the maximum Rate-of-Change-of-Frequency (ROCOF) limit is satisfied in response to a large generator outage. In contrast, the constraint priced in this paper, adopted from [2], ensures the frequency is arrested before the frequency reaches some critical frequency threshold. Although inertia and VI themselves are not capable of arresting frequency decline, the reserve requirement from [2] illustrates that inertia is capable of reducing the average ROCOF before the frequency nadir is reached, allowing other resources more time to react before the critical frequency threshold is met. We suggest that this is a more appropriate application for VI because IBRs are capable of reliably imitating inertia on the time scales associated with arresting frequency decline but may not be capable of reacting on faster time-scales required to reduce the maximum ROCOF.

Various works derive frequency response reserve requirements that ensure frequency is adequately arrested in response to a large generator outage; however, these works do not consider VI reserve, most do not consider FFR reserve as defined by ERCOT, and many do not derive prices for these reserve types in a market setting. References $[12,13$, 14] derive such reserve requirements using a model of frequency response reserve that is most consistent with our model of PFR reserve. Reference [15] uses a general model of a generator that is capable of accommodating FFR reserve and VI reserve; however, they utilize a pre-determined frequency trajectory that does not vary with the system operating point.
References $[16,17]$ claim to be easily generalized to accommodate multiple different models of frequency response reserve; however, they have only been studied with respect to a model that is most consistent with our model of PFR reserve, which experiences a time delay followed by a constant ramp rate. Reference [18] expands upon $[16,17]$ by deriving reserve prices for resources with different ramp rates and time delays. In contrast, our work models frequency response reserve types intended for IBRs as responding to the frequency during the post-outage dynamics rather than being a fixed function of time.

This paper differs from previous work by representing VI reserve in a reserve requirement that ensures frequency is adequately arrested in response to a large generator outage. Furthermore, this paper is the first to derive prices for primary frequency response reserve products having significantly different requirements for energy reserve procurement. Indeed, ERCOT currently requires FFR reserve to be sustained for 15 minutes after being deployed, which requires significant energy reserve [3] and may be burdensome for some IBRs such as storage devices. In contrast, the derivative response associated with VI reserve requires very little energy reserve because this response is only temporary. This allows us to study the incentives of IBRs with different power and energy reserve capabilities regarding which products they would provide most profitably. In this context we find that many IBRs will have incentive to provide both VI reserve and FFR reserve simultaneously. Furthermore, energy-constrained IBRs have incentive to provide more VI reserve than FFR reserve and power-constrained IBRs have incentive to provide more FFR reserve than VI reserve. Although IBRs generally could also provide PFR reserve, we show that they do not have incentive to do so because the PFR reserve price is always lower than the FFR reserve price.

The remainder of this paper is organized as follows. Section 2 describes the proposed frequency response products. Section 3 provides models for both profit maximizing market participants considered in our work, namely, IBRs and synchronous generators. Section 4 introduces VI reserve into the reserve requirement originally derived in $[2,8]$, places this requirement into a real-time co-optimization problem, derives the product prices, and proves that each IBR has incentive to follow its dispatched instructions. Section 5 provides numerical results. Section 6 concludes this paper.

\section{Frequency Response Products}

This section provides physical models of inertia, VI reserve, PFR reserve, and FFR reserve and 
also discusses the energy and power qualification requirements for an IBR to provide VI reserve, PFR reserve, and FFR reserve as a product in the electricity market. The general qualification requirement states that an IBR must have sufficient energy and power reserve to provide the reserve product of interest in response to a generator outage of size $L$. In fact, the reserve requirements in Section 4.1 ensure that the frequency remains above some critical frequency threshold $\underline{\omega}$ in response to a generator outage of size $L$, where $\underline{\omega}$ corresponds in practice to the threshold for involuntary under-frequency load shedding.

Throughout this paper we use subscripts to refer to elements of vectors and matrices. For example, $r_{i}$ is the $i^{\text {th }}$ element of vector $r$ and $H_{i j}$ is the element in the $i^{t h}$ row and $j^{t h}$ column of matrix $H$. The vector of ones is denoted $\mathbf{1}$ and the transpose operator is denoted $\dagger$.

\subsection{Swing Equation and Inertia}

Synchronous inertia, provided by synchronous generators, affects the frequency trajectory of the system. This trajectory is determined by the system dynamics and will be represented in this work by a simplified version of the swing equation. For simplicity, we will often refer to synchronous inertia as inertia and refer to synchronous generators as generators. The post-outage inertia provided by generator $i$ is fixed and will be denoted $M_{i}$ (in units of Watt-seconds or Ws).

The voltage frequency at time $t$ is modeled as being the same at each generator in the system and is denoted $\omega(t)$. The total post-outage inertia provided by synchronous generators is $\mathbf{1}^{\dagger} M$ and represents the sum of inertia values for all synchronous generators in service after the outage. The system dynamics are represented by the swing equation [19], which is expressed as follows:

$$
\frac{d \omega(t)}{d t}=\frac{\omega_{0}}{2\left(\mathbf{1}^{\dagger} M\right)}\left(\mathbf{1}^{\dagger} m(t)+\mathbf{1}^{\dagger} p(t)+\mathbf{1}^{\dagger} d(t)-e(t)\right),
$$

where $m(t) \in \mathbb{R}^{n}$ represents the vector of mechanical power output from the turbine governor of each generator in the system and $e(t) \in \mathbb{R}$ represents the total net electrical demand in the system. The net demand $e(t)$ will also include the nominal power trajectory of all IBRs. Deviation from the nominal trajectory provided by each IBR through VI and FFR are respectively denoted $p(t) \in \mathbb{R}^{\beta}$ and $d(t) \in \mathbb{R}^{\beta}$. The total number of generators and IBRs are respectively denoted $n$ and $\beta$. This model makes the simplifying assumption that there is no system damping. The nominal frequency is denoted $\omega_{0}$ and is assumed to be the frequency prior to the generator outage, which occurs at time $t=0$.

\subsection{Virtual Inertia Reserve}

The VI provided by inverter-based resource (IBR) $j$ intends to mimic inertia provided by synchronous generators and will be denoted $\hat{M}_{j}$. In order to provide VI, IBR $j$ must provide a required amount of VI reserve, which is denoted $\nu_{j}$ and represents a power quantity.

We define the VI response to be active only if the frequency is below its nominal value. This definition only requires energy reserve procurement in one direction consistent with current practices of most ISOs, which only define contingency related reserves to accommodate generator outages, not load outages.

A resource providing VI in the amount $\hat{M}_{j}$ will approximately provide the following power trajectory:

$$
p_{j}(t)= \begin{cases}\frac{-2 \hat{M}_{j}}{\omega_{0}} \frac{d \omega(t)}{d t} & \omega(t) \leq \omega_{0} \\ 0 & \omega(t)>\omega_{0}\end{cases}
$$

This response is equivalent to derivative control on the frequency with a proportionality constant $\frac{-2 \hat{M}_{j}}{\omega_{0}}$. Indeed, responding in this way requires a measurement of the derivative of the frequency $\frac{d \omega(t)}{d t}$. Since this value must be measured, VI does not respond instantaneously in the same way as inertia provided by synchronous generators. As a result, VI experiences a time delay as compared to inertia provided by synchronous generators meaning that (2) is only satisfied after a delay; however, this delay is very small and can be considered negligible for most applications except for mitigating the maximum ROCOF after a contingency.

In response to a large generator outage, the ROCOF will be largest the instant after the outage occurs and before any reserve types are capable of reacting, where the ROCOF is denoted $\left|\frac{d \omega(t)}{d t}\right|$ and $|\cdot|$ represents the absolute value. We assume that a ROCOF limit is enforced denoted $\bar{\omega}^{\prime}$, and effectively places a lower limit on the total post-outage synchronous inertia. A maximum available power requirement can then be determined from the VI response (2). Specifically, a unit providing VI must be able to provide the following amount of power reserve, termed VI reserve:

$$
\nu_{j}=\frac{2 \hat{M}_{j}}{\omega_{0}} \bar{\omega}^{\prime} .
$$

To follow the VI response in (2) an IBR must be capable of attaining a negative power response, e.g. $p_{j}(t)<0$. This negative power response will occur during small natural fluctuations in the frequency trajectory and when the frequency is being restored to its nominal value after an event. In this paper we assume that the negative power reserve procurement is negligibly small because the derivative of the frequency is typically small in 
magnitude during the recovery process after a frequency event. Future work may investigate an alternative power requirement for VI reserve in this direction.

A resource providing VI must have the ability to sustain its inertial response for a frequency deviation of $\omega_{0}-\underline{\omega}$. Integrating the VI response in (2) leads to the following energy requirement:

$$
\text { VIEnergyReserve }_{j}=\frac{2 \hat{M}_{j}\left(\omega_{0}-\underline{\omega}\right)}{\omega_{0}} .
$$

Importantly, a storage device providing VI reserve should be capable of reacting to multiple successive frequency events under the assumption that the frequency fully recovers to its nominal value between events. Specifically, the virtual inertia response (2) requires the energy reserve to be replenished between events when the frequency is restored to its nominal value so that sufficient energy would then be available to respond to a subsequent outage.

\subsection{Primary Frequency Response Reserve}

PFR reserve is compatible with conventional generator droop control provided by synchronous generators. Generator $i$ provides an amount of PFR reserve denoted $r_{i}$. We adopt the approximate piece-wise linear model of PFR reserve initially presented in [12] and explained in detail in [8]. This model of PFR reserve is similar to the constant ramp rate models in [12, 13, 14, 16, 17]. More specifically, after the frequency reaches the lower end of the frequency dead-band $\omega_{1}$ the turbine governor is modeled as experiencing a small time delay $\epsilon$. After this time delay, the mechanical power output $m_{i}(t)$ is modeled as having a constant governor ramp rate $\kappa_{i}$ that continues for a time of $\frac{r_{i}}{\kappa_{i}}$, allowing all PFR reserve to be deployed.

As we will see in Section 4.2, the price for FFR reserve is larger than the price for PFR reserve and thus IBRs do not have incentive to provide PFR reserve. Nevertheless, market rules do not prohibit IBRs from providing PFR reserve. If an IBR were to provide PFR reserve, then its energy requirement should be similar to the energy requirement for FFR reserve; however, this energy requirement is not specified in current protocols.

\subsection{Fast Frequency Response Reserve}

FFR reserve is defined consistently with ERCOT's definition [20, 21]. The amount of FFR reserve provided by IBR $j$ is denoted $b_{j}$. We assume that FFR reserve can be fully deployed instantaneously. The FFR reserve is deployed when the frequency drops below a frequency of $\omega_{2}<\omega_{1}$. That is, by design FFR deploys in ERCOT after the onset of PFR, assuming that the delay $\epsilon$ in activation of PFR is negligible. When deployed, FFR reserve instantaneously increases the quantity $d_{j}(t)$ by an amount $b_{j}$ for IBR $j$.

To provide FFR reserve, an IBR must meet a qualification requirement for power and for energy. First, the resource must be capable of deploying $b_{j}$ units of power and thus the power reserve requirement for FFR reserve is straight forward. Second, the resource must be capable of sustaining full deployment of $b_{j}$ for a specified amount of time $\Delta t$, which is set to 15 minutes, or $\Delta t=900$ seconds, in ERCOT [20].

$$
\text { FFREnergyReserve }_{j}=b_{j} \Delta t
$$

This requirement ensures that FFR reserve will last long enough for slower acting reserve to replace it.

\section{Profit Maximization}

We present a simplified myopic market setting that consists of two types of profit maximizing market participants. The first is a synchronous generator that provides electric generation, PFR reserve, and inertia as products in the electricity market. The second is an IBR that provides electric generation, FFR reserve, and VI reserve as products in the electricity market.

\subsection{Synchronous Generators}

We will assume that there is one generator located at each of the $n$ buses in the system without loss of generality. A synchronous generator $i$ is paid for producing a power quantity of $G_{i}$ in accordance with its Locational Marginal Price (LMP) $\pi_{i}$ and is paid for providing PFR reserve $r_{i}$ in accordance with the PFR reserve price $\chi$. A synchronous generator $i$ is also paid for providing a fixed amount of post-outage inertia $M_{i}$ in accordance with the price for inertia $\Psi$, see Remark 1. The LMP is specific to a location, or bus, in the system. The inertia price and PFR reserve price are common to all generators. The costs for generator $i$ are represented by the general convex function $C_{i}\left(G_{i}, r_{i}\right)$. Although the cost is generally represented as a function of the generation $G_{i}$ and reserve $r_{i}$, the cost of a synchronous generator typically only depends directly on the generation $G_{i}$. Under the assumption that the price is not affected by the generation and reserve quantities provided by a single generator, i.e. the price-taker assumption, each generator will maximize their profits by producing generation and reserve that solves the following problem.

$$
\begin{gathered}
\max _{r_{i} \in \mathbb{R}_{+}, G_{i} \in \mathbb{R}_{+}} \pi_{i} G_{i}+\chi r_{i}+\Psi M_{i}-C_{i}\left(G_{i}, r_{i}\right) \\
\underline{G}_{i} \leq G_{i} \leq \bar{G}_{i}-r_{i} \\
r_{i} \leq \bar{r}_{i}
\end{gathered}
$$


The generation limit constraint, which includes PFR reserve head-room, is represented in (6a). The constraint (6b) limits each generator's PFR reserve where the limit $\bar{r}_{i}$ is considered fixed from the perspective of the generator and is chosen by the ISO. In this context the PFR reserve limit $\bar{r}_{i}$ represents the maximum amount of PFR reserve a generator can provide before the frequency reaches the critical frequency threshold in response to a specified generator outage of size $L$, see Section 4.1. Any PFR reserve provided by generator $i$ that exceeds this value would be unavailable to arrest frequency decline due to ramping limitations. Intuitively, the ISO is only willing to pay for at most $\bar{r}_{i}$ units of PFR reserve from generator $i$ because ramping limitations physically limit the generator from providing more PFR reserve.

The PFR reserve limit $\bar{r}_{i}$ is an artifact of the reserve requirements proposed in Section 4.1, will be optimized as a variable in the real-time co-optimization problem (10), and is upper bounded by the offered PFR reserve capacity $\bar{R}_{i}$ by the constraint (10f). The offered PFR reserve capacity $\bar{R}_{i}$ is a quantity offered by each generator in a market setting and limits the PFR reserve a generator is willing to provide. ISO's typically place a hard limit on the offered PFR reserve capacity $\bar{R}_{i}$. In ERCOT the offered PFR reserve capacity $\bar{R}_{i}$ is typically limited by $0.2 \bar{G}_{i}$; however, this offered PFR reserve capacity limit depends on the generator's droop percentage, see [8].

Remark 1. The inertia for each generator is fixed and is therefore not represented as a variable in the profit maximization problem (6); however, the fixed inertia payment $\Psi M_{i}$ is included in the objective for completeness. Furthermore, the proposed reserve requirements aim to accommodate the simultaneous outage of the two generators with the largest capacity. In this context the two generators with the largest capacity have post-outage inertia levels of zero, e.g. $M_{i}=0$, and should not receive payment for providing inertia.

\subsection{Inverter-based Resources}

We assume there are $\beta$ IBRs in total located at various buses in the system. The sparse matrix $H \in \mathbb{R}^{n \times \beta}$ maps each IBR to its corresponding bus where IBR $j$ is located at bus $i$ if $H_{i j}=1$. IBR $j$ is paid for producing electric power $f_{j}$, which may be negative, based on the price $\pi^{\dagger} H_{j}$ where $H_{j}$ is the $j^{t h}$ column of $H$. The corresponding electric energy generation required from IBR $j$ is expressed as $\tau f_{j}$, where $\tau$ represents the market clearing interval length and is set to 5 minutes, or $\tau=300$ seconds, to match ERCOT's real-time market. IBR $j$ is paid for producing FFR reserve $b_{j}$ based on the price $\phi$ and paid for producing VI reserve $\nu_{j}$ based on the price $\psi$. These prices are modeled as being common to all IBRs. The costs for IBR $j$ are represented by the general convex function $P_{j}\left(f_{j}, b_{j}, \nu_{j}\right)$. Under the price-taker assumption, each resource will maximize its profits by producing generation and reserve that solves the following problem.

$$
\begin{gathered}
\max _{\substack{f_{j} \in \mathbb{R}, \nu_{j} \in \mathbb{R}_{+} \\
b_{j} \in \mathbb{R}_{+}}} \pi^{\dagger} H_{j} f_{j}+\phi b_{j}+\psi \nu_{j}-P_{j}\left(f_{j}, b_{j}, \nu_{j}\right) \\
\quad \underline{b}_{j} \leq f_{j} \leq \bar{b}_{j}-b_{j}-\nu_{j} \\
\underline{B}_{j} \leq \tau f_{j} \leq \bar{B}_{j}-\Delta t b_{j}-\frac{\left(\omega_{0}-\underline{\omega}\right)}{\bar{\omega}^{\prime}} \nu_{j}
\end{gathered}
$$

Constraint (7a) ensures that the electric power generation $f_{j}$ falls within the upper and lower power output limits, denoted $\underline{b}_{j}$ and $\bar{b}_{j}$, while also accounting for reserve head-room. Constraint (7b) ensures that the electric energy generation $\tau f_{j}$ falls within the upper and lower energy output limits, denoted $\underline{B}_{j}$ and $\bar{B}_{j}$, while also accounting for energy reserve. The energy reserve quantities for FFR reserve and virtual inertia are represented as linear expressions of the power quantities $b_{j}$ and $\nu_{j}$. The expression for FFR energy reserve $\Delta t b_{j}$ follows directly from equation (5). The expression for virtual inertia energy reserve $\frac{\left(\omega_{0}-\underline{\omega}\right)}{\bar{\omega}^{\prime}} \nu_{j}$ is obtained from equations (3) and (4). Specifically, the expression for virtual inertia $\hat{M}_{j}=\frac{\nu_{j} \omega_{0}}{2 \bar{\omega}^{\prime}}$ is obtained from (3) and substituted into (4). See Remark 2 for an example of an IBR that fits into this model.

Remark 2. The main body of this paper will leave the IBR profit maximization problem (7) general to accommodate many different types of IBRs and bidding strategies. However, the numerical results section, Section 5, will analyze an IBR that represents a storage device only participating in revenue streams associated with FFR reserve and VI reserve. This model will represent a storage device that does not participate in temporal energy arbitrage on the LMP and thus its power output will be fixed to $f_{j}=0$. In Section 5 , we also assume that the storage devices are fully charged, resulting in $\underline{b}_{j}=\underline{B}_{j}=0$. Furthermore, costs associated with FFR reserve and VI reserve will be approximated as being zero. Indeed, these costs should be low because the cost of charging the storage device to provide reserve can be amortized over many market intervals assuming that frequency events are rare.

\section{Product Pricing and Incentives}

This section proposes a reserve requirement that expands upon [2] and then places this reserve requirement into a real-time co-optimization problem. Prices are then defined for PFR reserve, FFR reserve, VI reserve and inertia products. We then provide a 
result stating that all market participants have incentive to follow their dispatched instructions from the ISO.

\subsection{Requirement to Arrest Frequency Decline}

Reference [2] derives reserve requirements that ensure sufficient frequency response to arrest the frequency decline before it reaches the critical frequency threshold of $\underline{\omega}$ in response to a generator outage of specified size $L$. To accommodate the simultaneous outage of the two largest generators in the system, $L$ would be chosen to be the sum of their capacities $\bar{G}_{i}$. This reserve requirement places a lower limit on the sum of all FFR reserve and PFR reserve and also places an upper limit on the amount of PFR reserve a single generator can provide. This PFR reserve limit is represented as a nonlinear function of the total FFR reserve $1^{\dagger} b$ and inertia including VI $\mathbf{1}^{\dagger} M+\mathbf{1}^{\dagger} \hat{M}$. The requirement is generally expressed as follows:

$$
\begin{gathered}
\mathbf{1}^{\dagger} r+\mathbf{1}^{\dagger} b \geq L \\
r \leq \kappa h\left(\mathbf{1}^{\dagger} M+\frac{\omega_{0}}{2 \bar{\omega}^{\prime}} \mathbf{1}^{\dagger} \nu, \mathbf{1}^{\dagger} b\right)+\delta
\end{gathered}
$$

The general requirement (8) ensures sufficient PFR reserve and FFR reserve to cover the loss of generation $L$. The rate-based PFR reserve limit (9) ensures that each generator has the ramping ability to provide all of its PFR reserve before the critical frequency threshold is reached in response to an outage of size $L$.

The rate-based PFR reserve limit (9) includes an offset constant $\delta \in \mathbb{R}^{n}$ that allows our main theoretical result to be more general. In the context of the reserve requirement from $[2,8]$, this offset constant can be assumed to be $\delta=\mathbf{0}$. The function $h(\cdot, \cdot)$ is derived in $[2,8]$, is continuously differentiable, strictly convex and increasing in both arguments. Intuitively this function represents the time taken to reach the frequency nadir after the generator outage occurs minus the PFR time delay so that multiplying $h(\cdot, \cdot)$ by the ramp rate $\kappa_{i}$ results in the maximum reserve generator $i$ can deploy by the time of the nadir. Notice that inertia provided by synchronous generators and VI reserve are both included in this requirement. In fact, we suggest that virtual inertia is capable of contributing to this requirement because it is capable of responding on time scales relevant to arresting frequency decline.

\subsection{Real-Time Co-Optimization}

We now extend the real-time co-optimization problems from $[2,8]$ by introducing VI reserve from Section 2.2, the IBR model from Section 3.2, and the reserve requirements from Section 4.1. The demand at node $i$ is assumed to be fixed at the value $D_{i}$.

$$
\begin{aligned}
& \min _{\substack{f \in \mathbb{R}^{\beta}, \nu \in \mathbb{R}_{+}^{\beta}, b \in \mathbb{R}_{+}^{\beta} \\
G \in \mathbb{R}_{+}^{n}, r \in \mathbb{R}_{+}^{n}, \bar{r} \in \mathbb{R}_{+}^{n}}} \sum_{i=1}^{n} C_{i}\left(G_{i}, r_{i}\right)+\sum_{j=1}^{\beta} P_{j}\left(f_{j}, b_{j}, \nu_{j}\right)(10) \\
& {[\lambda] \quad \mathbf{1}^{\dagger}(f+G-D)=0} \\
& {[\zeta] \quad S(H f+G-D) \leq \bar{F}} \\
& {[\mu] \quad L \leq \mathbf{1}^{\dagger} r+\mathbf{1}^{\dagger} b} \\
& {[\gamma] \quad \quad \bar{r} \leq \kappa h\left(\mathbf{1}^{\dagger} M+\frac{\omega_{0}}{2 \bar{\omega}^{\prime}} \mathbf{1}^{\dagger} \nu, \mathbf{1}^{\dagger} b\right)+\delta} \\
& {[\underline{\rho}, \bar{\rho}] \quad \underline{G} \leq G \leq \bar{G}-r} \\
& {[\underline{\sigma}, \bar{\sigma}] \quad r \leq \bar{r} \leq \bar{R}} \\
& {[\underline{\varrho}, \bar{\varrho}] \quad \underline{b} \leq f \leq \bar{b}-b-\nu} \\
& {[\underline{\varsigma}, \bar{\varsigma}] \quad \underline{B} \leq \tau f \leq \bar{B}-\Delta t b-\frac{(10 \mathrm{c})}{\bar{\omega}^{\prime}} \nu}
\end{aligned}
$$

Constraint (10a) enforces the overall system power balance. Constraint (10b) enforces $\ell$ transmission line limits, where $S \in \mathbb{R}^{\ell \times n}$ is a matrix of shift factors and $\bar{F} \in \mathbb{R}^{\ell}$ represents the maximum power flow on each transmission line. Constraint (10c) enforces the general reserve requirement from (8) and (10d) enforces the rate-based PFR reserve limit constraint from (9). Notice that the PFR reserve limit $\bar{r}_{i}$ is represented as a variable and is limited by the rate-based PFR reserve limit (10d), effectively limiting the PFR reserve $r_{i}$ indirectly. The constraints for each individual generator are represented by (10e) and (10f) and are identical to constraints (6a) and (6b) but also enforce the offered PFR capacity constraint $\bar{r} \leq \bar{R}$ as explained in Section 3.1. The constraints for each individual IBR are represented by $(10 \mathrm{~g})$ and (10h) and are identical to constraints (7a) and (7b). The Lagrange multipliers for each constraint are indicated on the left side in square brackets.

Remark 3. The real-time co-optimization problem (10) optimizes over the PFR reserve limit $\bar{r}_{i}$ for each generator $i$. Recall from Section 3.1 that the PFR reserve limit $\bar{r}_{i}$ is considered constant from the perspective of the generator. This is because the PFR reserve limit $\bar{r}_{i}$ only appears in the PFR reserve limit constraint (10d), and the right-hand-side of this constraint involves variables that are not controlled by the generator in a myopic real-time market context, namely, VI reserve $\nu$, FFR reserve $b$, and inertia $M$, which is assumed constant in the real-time market. Intuitively, generator $i$ exhibits ramping limitations preventing it from providing PFR reserve in excess of $\bar{r}_{i}$ in response to a generator outage of size $L$. Furthermore, the ISO is not willing to pay generator $i$ for providing excess PFR reserve that it is incapable of utilizing.

The proposed real-time co-optimization problem (10) is non-convex due to the non-linear constraints 
(10d). However, since the constraints are continuous, many iterative algorithms exist that typically converge to a point that solves the Karush-Kuhn-Tucker (KKT) conditions along with some Lagrange multipliers. In this paper we will assume that such a point exists for the real-time co-optimization problem (10) and can be obtained algorithmically using some iterative algorithm. This identified KKT point will represent the dispatched generation values, the dispatched reserve values, and the PFR reserve limit that is chosen by the ISO. The KKT point is defined as follows. We emphasize that we do not assume this KKT point is globally or even locally optimal for (10); however, practical iterative algorithms will typically converge to a KKT point that is at least locally optimal, see Remark 4.

Definition 1. The KKT conditions of the real-time co-optimization problem are solved by the point $\left(G^{\star}, r^{\star}, \bar{r}^{\star}, f^{\star}, b^{\star}, \nu^{\star}\right)$ along with the Lagrange multipliers $\left(\lambda^{\star}, \zeta^{\star}, \mu^{\star}, \gamma^{\star}\right)$, which are used in the proposed pricing structure, and the Lagrange multipliers $\left(\underline{\rho}^{\star}, \bar{\rho}^{\star}, \underline{\sigma}^{\star}, \bar{\sigma}^{\star}, \underline{\varrho}^{\star}, \bar{\varrho}^{\star}, \underline{\varsigma}^{\star}, \bar{\varsigma}^{\star}\right)$, which correspond to private constraints of the market participants.

Remark 4. The real-time co-optimization problems in $[2,8]$ do not consider VI reserve and are still non-convex due to the rate-based PFR reserve limit. These problems are also solved using iterative algorithms not guaranteed to converge to global optima.

\subsection{Product Prices}

Prices are defined by Lagrange multipliers of the constraints in the co-optimization problem as specified in Definition 1. The LMPs and the prices for the PFR reserve, FFR reserve, and VI reserve products are respectively as follows:

$$
\begin{aligned}
\pi_{i} & =-\lambda^{\star}-S_{i}^{\dagger} \zeta^{\star} \quad \forall i \in[1, \ldots, n] \\
\chi & =\mu^{\star} \\
\phi & =\mu^{\star}+\gamma^{\star \dagger} \kappa \nabla_{2} h \\
\psi & =\gamma^{\star \dagger} \kappa \nabla_{1} h \frac{\omega_{0}}{2 \bar{\omega}^{\prime}}
\end{aligned}
$$

where $\nabla_{1} h$ and $\nabla_{2} h$ represent the partial derivatives of $h(\cdot, \cdot)$ with respect to its first and second arguments evaluated at the point $\left(\mathbf{1}^{\dagger} M+\frac{\omega_{0}}{2 \bar{\omega}^{\prime}} \mathbf{1}^{\dagger} \nu^{\star}, \mathbf{1}^{\dagger} b^{\star}\right)$ and the $i^{\text {th }}$ column of $S$ is denoted $S_{i} \in \mathbb{R}^{\ell}$. Furthermore, since inertia and VI reserve have the same contribution to arresting frequency decline, their prices should agree in a way that is consistent with their relationship in (3). That is, the price for inertia is $\Psi:=\frac{2 \bar{\omega}^{\prime}}{\omega_{0}} \psi$.

The FFR price upper bounds the PFR price because the second term on the RHS of equation (13) is non-negative. This pricing structure effectively places more value in FFR reserve due to its fast ramping capabilities. Furthermore, the energy requirement is similar for PFR reserve and FFR reserve. As a result, an IBR will always prefer to provide FFR reserve over PFR reserve.

\subsection{Incentive Alignment}

With the prices set as in (11)-(14), the generation and reserve quantities from Definition 1 solve the individual profit maximization problems for generators and IBRs, as defined in Section 3. Intuitively, this is because all non-linear constraints in the real-time co-optimization problem are being priced and because the individual profit maximization problems are convex. This is stated formally in the following theorem.

Theorem 1. Consider a KKT point as in Definition 1 and assume the prices are set as in (11)-(14).

(a) The generation dispatch and reserve quantities $\left(G_{i}^{\star}, r_{i}^{\star}\right)$ solve the generator profit maximization problems (6) for each generator $i$.

(b) The generation dispatch and reserve quantities $\left(f_{j}^{\star}, b_{j}^{\star}, \nu_{j}^{\star}\right)$ solve the IBR profit maximization problems (7) for each IBR $j$.

Proof: If the the KKT point from Definition 1 solves the KKT conditions for the problems (6) and (7), then this point must also be globally optimal for the problems (6) and (7) because they are both convex and thus the KKT conditions imply global optimality. To complete the proof, we prove that the KKT point from Definition 1 satisfies the KKT conditions for problems (6) and (7). Note that there are three parts to the KKT conditions: the stationarity condition, the dual feasibility condition, and the primal feasibility condition.

(a) The KKT point from Definition 1 must satisfy the following stationarity condition for problem (10) with respect to $G_{i}$ and $r_{i}$ respectively:

$$
\begin{aligned}
& \nabla_{1} C_{i}+\lambda^{\star}+S_{i}^{\dagger} \zeta^{\star}-\underline{\rho}_{i}^{\star}+\bar{\rho}_{i}^{\star}=0 \\
& \nabla_{2} C_{i}-\mu^{\star}+\bar{\rho}_{i}^{\star}+\underline{\sigma}_{i}^{\star}=0
\end{aligned}
$$

where $\nabla_{1} C_{i}$ and $\nabla_{2} C_{i}$ represent partial derivatives of $C_{i}(\cdot, \cdot)$ with respect to its first and second arguments evaluated at $\left(G_{i}^{\star}, r_{i}^{\star}\right)$ and are assumed to be continuous.

If the prices are chosen as in (11) and (12), then these conditions are identical to the stationarity conditions of (6) with respect to $G_{i}$ and $r_{i}$ respectively where $\left[\rho_{i}^{\star}, \bar{\rho}_{i}^{\star}\right]$ represent the Lagrange multipliers of constraints (6a) and $\underline{\sigma}_{i}^{\star}$ represents the Lagrange multiplier of the constraint (6b). Furthermore, these Lagrange multipliers must be non-negative in order to satisfy the dual feasibility condition for the co-optimization problem (10) and thus they must also satisfy the dual 
feasibility condition for (6). Finally, since primal feasibility is satisfied for problem (10) the values $\left(G_{i}^{\star}, r_{i}^{\star}\right)$ must satisfy constraints (10e) and (10f) and as a result must also satisfy constraints (6a) and (6b), proving primal feasibility for (6). As a result, the KKT conditions hold for (6), proving part (a).

(b) The KKT point from Definition 1 must satisfy the following stationarity condition for problem (10) with respect to $f_{j}, b_{j}$, and $\nu_{j}$ respectively:

$$
\begin{aligned}
& \nabla_{1} P_{j}+\lambda+H_{j}^{\dagger} S^{\dagger} \zeta^{\star}-\underline{\varrho}_{j}^{\star}+\bar{\varrho}_{j}^{\star}-\tau \underline{\varsigma}_{j}^{\star}+\tau \bar{\varsigma}_{j}^{\star}=0 \\
& \nabla_{2} P_{j}-\mu^{\star}-\gamma^{\star \dagger} \kappa \nabla_{2} h+\bar{\varrho}_{j}^{\star}+\bar{\varsigma}_{j}^{\star} \Delta t=0 \\
& \nabla_{3} P_{j}-\gamma^{\star \dagger} \kappa \nabla_{1} h \frac{\omega_{0}}{2 \bar{\omega}^{\prime}}+\bar{\varrho}_{j}^{\star}+\bar{\varsigma}_{j}^{\star} \frac{\left(\omega_{0}-\underline{\omega}\right)}{\bar{\omega}^{\prime}}=0
\end{aligned}
$$

where $\nabla_{1} P_{j}, \quad \nabla_{2} P_{j}$, and $\nabla_{3} P_{j}$ represent partial derivatives of $P_{j}(\cdot, \cdot, \cdot)$ with respect to its first, second, and third arguments evaluated at $\left(f_{j}^{\star}, b_{j}^{\star}, \nu_{j}^{\star}\right)$ and are assumed to be continuous.

If the prices are chosen as in (11), (13), and (14), then these conditions are identical to the stationarity conditions of (7) with respect to $f_{j}, b_{j}$, and $\nu_{j}$ respectively where $\left[\underline{\varrho}_{j}^{\star}, \bar{\varrho}_{j}^{\star}\right]$ represent the Lagrange multipliers of constraints (7a) and $\left[\varsigma_{j}^{\star}, \bar{\varsigma}_{j}^{\star}\right]$ represent the Lagrange multipliers of constraints (7b). Furthermore, these Lagrange multipliers must be non-negative in order to satisfy the dual feasibility condition for the co-optimization problem (10) and thus they must also satisfy the dual feasibility condition for (7). Finally, since primal feasibility is satisfied for problem (10) the values $\left(f_{j}^{\star}, b_{j}^{\star}, \nu_{j}^{\star}\right)$ must satisfy constraints $(10 \mathrm{~g})$ and (10h) and as a result must also satisfy constraints (7a) and (7b), proving primal feasibility for (7). As a result, the KKT conditions hold for (7), proving part (b).

\section{Numerical Results}

This section studies the coupling between PFR reserve, FFR reserve, VI reserve and inertia in a market setting using a 2000 bus test case that is intended to have characteristics similar to the ERCOT system [22]. The PFR reserve costs of a synchronous generator are assumed to be zero, consistent with Section 3.1, resulting in cost functions that are only a function of generation and represent the hourly cost of generating $G_{i} \mathrm{MW}$ of power. The 75 natural gas generators with the largest capacity are selected to provide PFR reserve and their offered PFR capacity is $\bar{R}_{i}=0.2 \bar{G}_{i}$. We consider a loss of generation of $L=2750 \mathrm{MW}$ and an FFR duration requirement of $\Delta t=900$ seconds unless stated otherwise. The function $h(\cdot, \cdot)$ is defined using parameters from [2]. Although ERCOT does not currently enforce a ROCOF limit, we assume it is $\bar{\omega}^{\prime}=0.5 \mathrm{~Hz} / \mathrm{s}$ and assume the post-outage inertia is $163 \mathrm{GWs}$. We use Matlab and Knitro's interior-point solver [23].

We assume each IBR $j$ represents a fully charged storage device as described in Remark 2 and has power capacity $\bar{b}_{j}=1 \mathrm{MW}$. Consistent with Remark 2 this storage device does not participate in temporal energy arbitrage and thus $f_{j}$ is set to zero. As a result, these storage devices do not appear in the transmission constraints in the co-optimization problem (10) and their physical location in the system is not modeled. For this reason we do not specify the location of the IBRs. To emulate the diversity of typical market participants, the energy capacity is chosen randomly for each IBR. Specifically, the ratio of energy to power capacity $\bar{B}_{j} / \bar{b}_{j}$ is uniformly randomly sampled between the values of 100 and 1000 seconds, which represents approximate power to energy capacity ratios for lithium ion batteries [24]. In other words, the MWs energy capacity is modeled as $\bar{B}_{j} \sim U[100,1000]$.

Figure 1 illustrates the reserve procurement, the prices, and the payments as the number of IBRs increase from 0 to 1000 , which increases the total MW capacity of IBRs $1^{\dagger} \bar{b}$ from 0 to 1000 MW. Figure 1a provides the total PFR reserve, FFR reserve, and VI reserve as the total power capacity $\mathbf{1}^{\dagger} \bar{b}$ increases. FFR reserve and VI reserve increase linearly with the total power capacity with less total reserve being designated for VI reserve. As FFR reserve increases it directly replaces PFR reserve in order to strictly satisfy the general requirement $(10 \mathrm{c})$.

Figure $1 \mathrm{~b}$ provides the prices for PFR reserve, FFR reserve, VI reserve, and the LMP, where the LMP $\pi_{i}$ is the same at each bus $i$ in the system because there is no transmission congestion. Consistent with current practice, these prices are specified in units of $\$ / \mathrm{MWh}$, which represents the price of providing a MW quantity of generation or reserve for an entire hour. The price for inertia $\Psi$ is not provided in this plot; however, it is proportional to the VI reserve price $\psi$ with proportionality constant $\frac{2 \bar{\omega}^{\prime}}{\omega_{0}} \approx 0.0167 \mathrm{~s}^{-1}$, as explained in Section 4.3. All prices steadily decline as more IBRs are introduced. As expected from Section 4.3, the FFR reserve price $\phi$ is always higher than the PFR reserve price $\chi$. As explained in Section 2.3, PFR reserve and FFR reserve have a similar energy reserve requirements and thus IBRs do not have incentive to provide PFR reserve. Similarly, the VI reserve price is always lower than the FFR reserve price. However, IBRs may have incentive to provide VI reserve as opposed to FFR reserve because VI reserve has a much lower energy reserve requirement as enforced in (7b). Specifically, the FFR energy reserve required to provide a single unit of FFR power reserve is $\frac{\Delta t \bar{\omega}^{\prime}}{\left(\omega_{0}-\underline{\omega}\right)}=750$ times larger than 


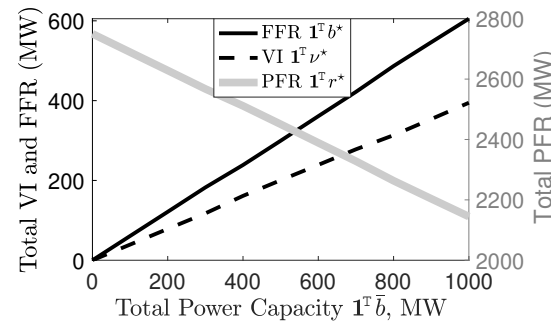

(a) Total Reserve Procurement

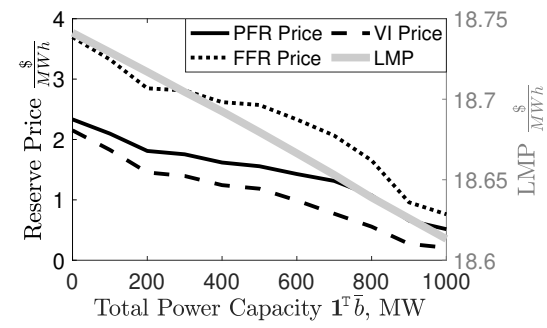

(b) Prices

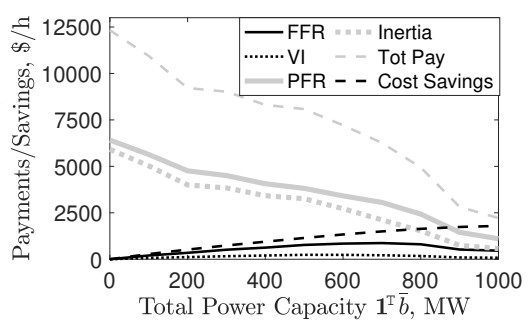

(c) Payments and Cost Savings

Figure 1: Three plots illustrate the effect of increasing the total number of IBRs. (a) Total power reserve provided for VI reserve, FFR reserve, and PFR reserve. (b) Reserve prices and the LMP, where the LMP is the same at each bus. (c) Total payments for each primary frequency response market product as well as the total generation cost savings.

the VI energy reserve required to provide a single unit of VI power reserve.

Figure 1c provides the payments for all primary frequency response products over an hour long period. For each total power capacity $\mathbf{1}^{\dagger} \bar{b}$ the order from highest to lowest paid product is as follows: PFR reserve, inertia, FFR reserve, and VI reserve. FFR reserve and VI reserve receive zero payment when the total power capacity is $\mathbf{1}^{\dagger} \bar{b}=0$ and this payment begins to increase as their product quantities increase. However, this trend ends when the total power capacity $\mathbf{1}^{\dagger} \bar{b}$ reaches a quantity of approximately $700 \mathrm{MW}$, at which point the decreasing FFR reserve price and VI reserve price cause their total payments to decrease despite their increasing product quantity. Furthermore, PFR reserve and synchronous inertia receive large payment when the total power capacity $\mathbf{1}^{\dagger} \bar{b}$ is low. This is when the reserve requirements are most constrained, prices are highest, and little FFR reserve and VI reserve are available.

Figure 1c illustrates the benefit of introducing the reserve types that are intended for IBRs, namely, VI reserve and FFR reserve. Introducing these products into the market significantly reduces total reserve payments and total generation costs. In our example, when total IBR power capacity is $\mathbf{1}^{\dagger} \bar{b}=0 \mathrm{MW}$ the total PFR reserve payments are $6414 \$ / \mathrm{h}$ and the total inertia payments are $5915 \$ / \mathrm{h}$. When $\mathbf{1}^{\dagger} \bar{b}=1000 \mathrm{MW}$ the total payments for all reserve products and inertia sum to only $2215 \$ / \mathrm{h}$ and the total generator costs reduce by $1795 \$ / \mathrm{h}$, as represented by the trajectory labeled cost savings.

Finally, we illustrate the incentives seen by an IBR that experiences a trade-off between two revenue streams associated with FFR reserve and VI reserve. Figure 2 plots each IBR's optimal VI reserve to FFR reserve ratio $\nu_{j}^{\star} / b_{j}^{\star}$ versus its energy capacity to power capacity ratio $\bar{B}_{j} / \bar{b}_{j}$ for different values of $\Delta t$. For a given $\Delta t$, one point in this plot represents one of the 1000 IBRs that were introduced. Throughout our testing, we numerically observed that the VI reserve to FFR reserve ratio $\nu_{j}^{\star} / b_{j}^{\star}$ for any given IBR $j$ as determined by the co-optimization problem does not change as the total power capacity $1^{\dagger} \bar{b}$ increases between the values of 0 to $1000 \mathrm{MW}$. As a result, these curves remain the same for any total power capacity $\mathbf{1}^{\dagger} \bar{b}$ between the values of 0 to $1000 \mathrm{MW}$. Furthermore, the value $\Delta t=900$ seconds matches that used in Figure 1 .

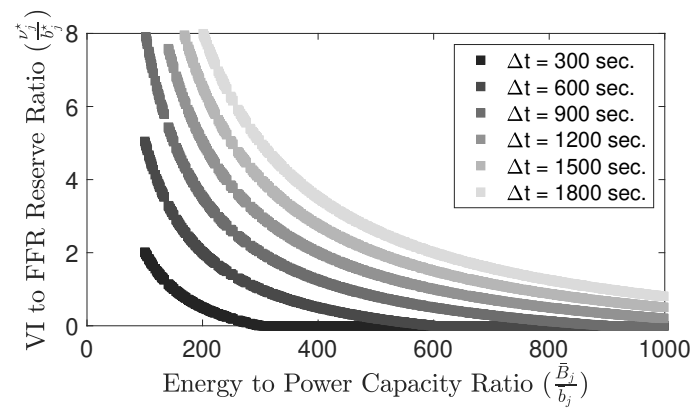

Figure 2: Each IBR's optimal VI reserve to FFR reserve ratio $\nu_{j}^{\star} / b_{j}^{\star}$ versus its energy capacity to power capacity ratio $\bar{B}_{j} / \bar{b}_{j}$ for different values of $\Delta$.

Figure 2 shows that the optimal VI reserve to FFR reserve ratio $\nu_{j}^{\star} / b_{j}^{\star}$ for an IBR decreases in its energy capacity to power capacity ratio $\bar{B}_{j} / \bar{b}_{j}$. In other words, an IBR with a small energy capacity and a large power capacity will provide more VI reserve than FFR reserve. Conversely, an IBR with a large energy capacity and a small power capacity will provide more FFR reserve than VI reserve. Furthermore, the optimal VI reserve to FFR reserve ratio $\nu_{j}^{\star} / b_{j}^{\star}$ reaches zero at the point where $\bar{B}_{j} / \bar{b}_{j}=\Delta t$. Intuitively, if an IBR had unlimited energy capacity it would have incentive to provide FFR reserve as opposed to VI reserve because the FFR reserve price is higher; however, the energy capacity constraint (7b) may prevent the IBR from allocating all of its power capacity towards FFR reserve. If the energy capacity to power capacity ratio exceeds $\bar{B}_{j} / \bar{b}_{j}=\Delta t$, then all power capacity can be allocated towards FFR reserve without reaching the energy capacity. 
Figure 2 also illustrates the effect of varying the value $\Delta t$, which represents the required amount of time an IBR must be capable of providing a full FFR response, see Section 2.4. In fact, an ISO can design this parameter to adjust the incentives of an IBR. Specifically, as $\Delta t$ increases, each IBR will have incentive to provide less FFR reserve as compared to VI reserve, moving the curve up and to the right. However, this effect diminishes as $\Delta t$ increases, illustrated by each successive curve shifting by a smaller amount.

\section{Conclusions}

This paper derived prices for PFR reserve, FFR reserve, VI reserve, and inertia as products in a real-time electricity market. The prices were derived from Lagrange multipliers of a reserve requirement that ensures frequency decline will be arrested before reaching a critical frequency threshold in response to a specified generator outage. We proved that the proposed prices provide incentive for IBRs to follow their dispatch instructions. We also determined that power-constrained IBRs prefer to provide FFR reserve over VI reserve and energy-constrained IBRs prefer to provide VI reserve over FFR reserve. Finally, we illustrated that the introduction of FFR reserve and VI reserve reduce total reserve payments and total generation costs.

\section{References}

[1] NERC, "Fast frequency response concepts and bulk power system reliability needs," tech. rep., North American Electric Reliability Corporation, March 2020.

[2] M. Garcia and R. Baldick, "Real-time co-optimization: Interdependent reserve types for primary frequency response," in Proceedings of the Tenth ACM International Conference on Future Energy Systems, pp. 550-555, ACM, 2019.

[3] P. Du et al., "New ancillary service market for ERCOT," IEEE Access, vol. 8, pp. 178391-178401, 2020.

[4] D. Fernández-Muñoz, J. I. Pérez-Díaz, I. Guisández,

M. Chazarra, and Á. Fernández-Espina, "Fast frequency, control ancillary services: An international review," Renewable and Sustainable Energy Reviews, vol. 120, p. 109662, 2020.

[5] P. Mancarella et al., "Power system security assessment of the future national electricity market," University of Melbourne, 2017.

[6] ERCOT, "NPRR 581: Add fast responding regulation service as a subset of regulation service," tech. rep., ERCOT, February 2014.

[7] W. Li, P. Du, and N. Lu, "Design of a new primary frequency control market for hosting frequency response reserve offers from both generators and loads," IEEE Transactions on Smart Grid, vol. 9, no. 5, pp. 4883-4892, 2018.

[8] M. Garcia and R. Baldick, "Requirements for interdependent reserve types providing primary frequency control," IEEE Transactions on Power Systems, 2021.

[9] U. Tamrakar et al., "Virtual inertia: Current trends and future directions," Applied Sciences, vol. 7, no. 7, p. 654, 2017.

[10] B. K. Poolla, S. Bolognani, L. Na, and F. Dörfler, "A market mechanism for virtual inertia," IEEE Transactions on Smart Grid, 2020.

[11] M. Paturet et al., "Economic valuation and pricing of inertia in inverter-dominated power systems," arXiv preprint arXiv:2005.11029, 2020.

[12] H. Chávez, R. Baldick, and S. Sharma, "Governor rate-constrained OPF for primary frequency control adequacy," IEEE Transactions on Power Systems, vol. 29, no. 3, pp. 1473-1480, 2014.

[13] V. Trovato, A. Bialecki, and A. Dallagi, "Unit commitment with inertia-dependent and multispeed allocation of frequency response services," IEEE Transactions on Power Systems, vol. 34, no. 2, pp. 1537-1548, 2018.

[14] L. Badesa, F. Teng, and G. Strbac, "Optimal portfolio of distinct frequency response services in low-inertia systems," IEEE Transactions on Power Systems, vol. 35, no. 6, pp. 4459-4469, 2020.

[15] L. E. Sokoler, P. Vinter, R. Bærentsen, K. Edlund, and J. B. Jørgensen, "Contingency-constrained unit commitment in meshed isolated power systems," IEEE Transactions on Power Systems, vol. 31, no. 5, pp. 3516-3526, 2015.

[16] L. Badesa, F. Teng, and G. Strbac, "Conditions for regional frequency stability in power system scheduling_part I: Theory," IEEE Transactions Power Systems, 2021.

[17] L. Badesa, F. Teng, and G. Strbac, "Conditions for regional frequency stability in power system scheduling-part II: Application to unit commitment," IEEE Transactions Power Systems, 2021.

[18] L. Badesa, F. Teng, and G. Strbac, "Pricing inertia and frequency response with diverse dynamics in a mixed-integer second-order cone programming formulation," Applied Energy, vol. 260, p. 114334, 2020.

[19] P. M. Anderson and A. A. Fouad, Power system control and stability. John Wiley \& Sons, 2008.

[20] ERCOT, "NPRR 863: Creation of primary frequency response service product and revisions to responsive reserve," tech. rep., ERCOT, January 2018.

[21] C. Liu and P. Du, "Participation of load resources in day-ahead market to provide primary-frequency response reserve," IEEE Transactions on Power Systems, vol. 33, no. 5, pp. 5041-5051, 2018.

[22] A. B. Birchfield, T. Xu, K. M. Gegner, K. S. Shetye, and T. J. Overbye, "Grid structural characteristics as validation criteria for synthetic networks," IEEE Transactions power systems, vol. 32, no. 4, pp. 3258-3265, 2017.

[23] R. H. Byrd, J. Nocedal, and R. A. Waltz, "Knitro: An integrated package for nonlinear optimization," in Large-scale nonlinear optimization, pp. 35-59, Springer, 2006.

[24] M. J. Lain, J. Brandon, and E. Kendrick, "Design strategies for high power vs. high energy lithium ion cells," Batteries, vol. 5, no. 4, p. 64, 2019. 